\title{
Folate-vitamin $B_{12}$ interrelationships in the central nervous system
}

\author{
BY JOHN M. SCOTT \\ Department of Biochemistry, Trinity College, Dublin 2, Republic of Ireland
}

Vitamin $B_{12}$ deficiency in man manifests itself clinically as an anaemia and a neuropathy.

\section{WHAT CAUSES THE ANAEMIA?}

The most accepted explanation as to how the anaemia arises is that vitamin $B_{12}$ deficiency interferes with the functioning of the folate cofactors as put forward by Herbert \& Zalushy (1962) and commonly called the 'methyl trap hypothesis'. The folates normally function as coenzymes that accept so-called $C_{1}$ units, which are transferred to them by enzymes involved in the degradation of histidine, formate, sarcosine and glycine or by the enzyme serine hydroxymethyltransferase $(E C 2.1 .2 .1)$ which converts serine to glycine. This last reaction is quantitatively and functionally the most important, particularly in replicating cells. The $\mathrm{C}_{1}$ cofactors produced in this and the other reactions are then used in biosynthetic reactions. Thus, 10 -formyltetrahydrofolate is used as a cofactor for two of the enzymes involved in purine biosynthesis, while 5,10-methylenetetrahydrofolate is involved in pyrimidine biosynthesis (Fig. 1). Apart from this role in the biosynthesis of purines and pyrimidines and, thus, DNA and RNA, the folates provide a source of methyl groups for dozens of methyltransferase enzymes. This is done by converting 5,10-methylenetetrahydrofolate to 5-methyltetrahydrofolate with this latter being used to methylate homocysteine to methionine by the vitamin $\mathrm{B}_{12}$-dependent enzyme methionine synthase ( $E C$ 2.1.1.13) (Fig. 1).

This methionine can then be activated with ATP to produce S-adenosylmethionine (SAM) which in turn can donate its methyl group to a methyltransferase giving a methylated product and S-adenosylhomocysteine (SAH). Normally in cells this SAH is not permitted to accumulate and is enzymically hydrolysed to homocysteine. This can then be recycled back to methionine and SAM via methionine synthase with a new methyl group which would be donated by 5-methyltetrahydrofolate.

The methyl trap hypothesis suggests that the enzymic conversion of 5,10 methylenetetrahydrofolate to 5-methyltetrahydrofolate is irreversible in vivo and that once this latter cofactor is formed it requires a functioning methionine synthase for it to become available again as part of the pool of other folate cofactors. It is suggested that in vitamin $B_{12}$ deficiency or when the enzyme methionine synthase is inactivated by, for example, the anaesthetic gas nitrous oxide (see p. 221), the cellular folate cofactors become metabolically trapped as 5-methyltetrahydrofolate. The inability of a cell to demethylate this cofactor results in its accumulation at the expense of those forms used in DNA and RNA biosynthesis. Thus, such cells would be in a sort of pseudo folatedeficient state most obviously seen as an anaemia. It might at first seem improbable that cells would so readily and foolishly participate in their own destruction. However, this was resolved by the finding by Kutzbach \& Stokstad (1967) that the synthesis of 5 -methyltetrahydrofolate in cells is controlled by the level of SAM. Falling levels of SAM, as might be expected to occur when the activity of methionine synthase is 


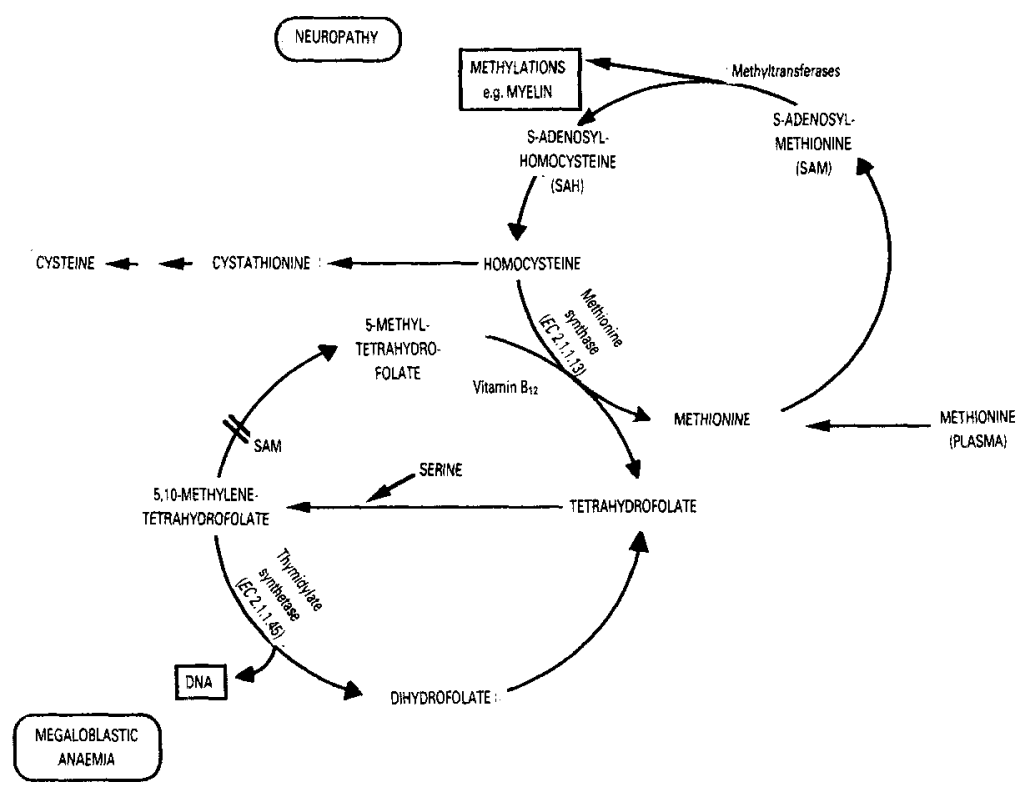

Fig. 1. The role of folate and vitamin $B_{12}$ in channelling $C_{1}$ units from serine to DNA biosynthesis or to provide methyl groups used in the biosynthesis of myelin and other brain structures.

diminished as in vitamin $B_{12}$ deficiency, result in an increase in the activity of 5,10 -methylenetetrahydrofolate reductase ( $E C$ 1.7.99.5), which in turn increases synthesis of 5-methyltetrahydrofolate. Thus, vitamin $\mathrm{B}_{12}$-deficient cells misread the dropping levels of methionine and SAM as a need to divert more of the folate cofactors into the methyl trap, thus exacerbating the situation.

There are still a number of questions about the validity of the methyl trap hypothesis, e.g. cellular replication in some species is not affected by vitamin $B_{12}$ deficiency and not all the cellular folates are in the trap. These and other concerns have led Chanarin et al. (1980) to put forward an alternative hypothesis called the formate starvation hypothesis. A function of SAM other than for methylation reactions is that it participates in the biosynthesis of polyamines and this also produces formate which they claim is used to convert tetrahydrofolate into formyltetrahydrofolate. When methionine synthase activity is decreased with a consequent decrease in SAM and the formate derived from it, they suggest that formyltetrahydrofolates are not formed. This, it is suggested, results in disruption of cellular folate metabolism since the formyl derivatives appear to be the preferred substrates for synthesis of the important cellular folate polyglutamates. When it was shown that administration of formate did not reverse the effects of vitamin $B_{12}$ deficiency (Keating et al. 1983), they suggested that this is because the formate involved is 'active formate' (Chanarin, 1990). The methyl trap hypothesis gives a good basis for explaining the clinical aspects of the megaloblastic anaemia of vitamin $\mathbf{B}_{12}$ deficiency and this is also true for the clinical sequela seen in inborn errors of enzymes related to this 
area. One would expect an anaemia which would be clinically indistinguishable from that found in folic acid deficiency. That folic acid can treat the anaemia could be predicted since initially it will form tetrahydrofolate which is not yet in the trap. There are now some dozens of cases of an inborn error where 5,10-methylenetetrahydrofolate reductase activity is diminished. None of these patients develop anaemia (Erbe, 1986). By contrast patients with inborn errors of metabolism that indirectly or directly affect methionine synthase activity all develop anaemia. The latter would cause trapping of 5methyltetrahydrofolate. While reductase deficiency might and indeed does give rise to other clinical features (see p. 223), it would not cause trapping of 5-methyltetrahydrofolate (Fig. 1).

\section{WHAT CAUSES THE NEUROPATHY?}

Because the anaemia and the neuropathy are often present completely independently of each other and because the former is treated by the administration of folic acid, while such therapy masks or exacerbates the neuropathy, it was traditionally accepted that each was due to deficiency of different vitamin $B_{12}$ functions. This fitted nicely with the knowledge that there are two and apparently only two vitamin $\mathrm{B}_{12}$-dependent enzymes in mammalian cells; methionine synthase and methylmalonyl-CoA mutase (EC 5.4.99.2).

It was known that decreased activity of the mutase leads to accumulation of methylmalonyl-CoA which is seen as its degradation product methylmalonate in the plasma and urine of vitamin $B_{12}$-deficient patients. It was suggested that high levels of the former caused it to be inappropriately used for fatty acid biosynthesis instead of acetyl-CoA (Frankel, 1973), and that myelin synthesized with these abnormal fatty acids was unstable and degraded. The finding of abnormal odd-chain fatty acids in myelin isolated from vitamin $B_{12}$-deficient animals and from the peripheral nerves of similarly deficient human subjects gave experimental support for this hypothesis.

The function of the synthase in providing methyl groups for methyltransferases was, of course, well established at a biochemical level and it was also known that SAMdependent methyltransferases were involved in the methylation of arginine residues of myelin basic protein (Baldwin \& Carnegie, 1971). However, the importance of methionine synthase in regenerating SAM was not really appreciated. It was known that methionine being an essential amino acid was present in excess in most human and animal diets. There was little to suggest that it, and with it SAM, would become limiting even in vitamin $B_{12}$ deficiency. In fact this probably is the case in the liver where catabolism of the excess methionine which takes place via SAM and betaine, a degradation product of choline, represents a vitamin $\mathrm{B}_{12}$-independent mechanism for recycling cellular homocysteine back to methionine. However, vitamin $B_{12}$ deficiency in neural tissue could be a completely different problem. First, there is a complete absence of the enzyme betaine-homocysteine methyltransferase (EC 2.1.1.5; McKeever et al. 1991 ) and second, unlike liver, neural tissue was probably never intended to take up and catabolize large amounts of methionine.

Beginning in 1980 a series of experiments emerged that made us and others re-evaluate deficiency of the mutase as the cause of the neuropathy. It had been found that humans exposed to the anaesthetic gas $\mathrm{N}_{2} \mathrm{O}$ developed a megaloblastic bone marrow and that this was due to inactivation of methionine synthase (Amess et al. 1978). This was quickly 
applied to animal studies since it had always been very difficult to deplete methionine synthase activity in vivo in experimental animals by feeding vitamin $\mathrm{B}_{12}$-deficient diets.

While $\mathrm{N}_{2} \mathrm{O}$ treatment of rodents gave biochemical results similar to those found previously in vitamin $\mathrm{B}_{12}$-deficient animals, i.e. decreased retention of 5-methyltetrahydrofolate by the liver (McGing et al. 1978) and decreased methionine synthase activity (Deacon et al. 1980), no signs or symptoms of the anaemia or the neoropathy were apparent in the vitamin $\mathrm{B}_{12}$-deficient or $\mathrm{N}_{2} \mathrm{O}$-treated rat (Scott et al. 1979).

In 1980 our group treated monkeys with $\mathrm{N}_{2} \mathrm{O}(150 \mathrm{ml} / \mathrm{l})$ for periods of weeks. None of the four monkeys involved in this initial study developed an anaemia or even megaloblastic changes in the bone marrow (Dinn et al. 1980). However, they did develop ataxia and on post-mortem examination were found to have peripheral neuropathy, Wallerian changes and sub-acute combined degeneration, i.e. similar pathology to that found previously in post-mortem sections from patients with pernicious anaemia.

It was suggested (Scott \& Weir, 1981) that inability to regenerate methionine from homocysteine in nerve tissue of such animals would lead to an inadequate supply of methionine and with it SAM and that this caused demyelination or dysmyelination. Experimental evidence to this end was furnished in the demonstration that monkeys kept in $\mathrm{N}_{2} \mathrm{O}$ for periods of weeks developed ataxia and neuropathy but that paired monkeys in the same environment given large oral doses of methionine daily showed no signs of clinical ataxia (Scott et al. 1981). Further it was found in the same study that post-mortem examination of the cord showed that those monkeys given methionine had demyelination judged to be about half that of the unprotected animals. Direct measurement of the levels of SAM present in the neural tissue of these animals was not attempted. Subsequently other investigators found that the levels of SAM in the brain and spinal cords of $\mathrm{N}_{2} \mathrm{O}$-treated rats (Lumb et al. 1983) and fruit bats (Rousettus aegypticus; Van der Westhuyzen \& Metz, 1983) were not significantly reduced when compared with normal controls that had not undergone $\mathrm{N}_{2} \mathrm{O}$ treatment. While the former species does not get the neuropathy on exposure to $\mathrm{N}_{2} \mathrm{O}$, the latter does and it was, thus, suggested by these groups that the neuropathy was not due to diminished SAM or caused by hypomethylation of myelin and other neural structures.

At that time we had succeeded in producing the ataxia and the neuropathy in the pig (Weir et al. 1988). As with the monkey high levels of dietary methionine reduced the ataxia and the myelopathy. We found that $\mathrm{N}_{2} \mathrm{O}$ treatment, as in the rat and fruit bat, did not significantly reduce the level of SAM. What was significant was that there was a marked elevation in the level of SAH. The activity of methyltransferase is tightly regulated in vivo not by the tissue levels of SAM but by the ratio of this cofactor to the form produced after methylation, i.e. SAH (Hoffman et al. 1980). This is often called the 'methylation ratio'. Thus, in the brain and spinal cord of pigs treated with $\mathrm{N}_{2} \mathrm{O}$ it was clear that a reduced methylation ratio existed which would be expected to inhibit neural methyltransferase in vivo (Weir et al. 1988). While high levels of SAH were also present in the $\mathrm{N}_{2} \mathrm{O}$-treated pigs given dietary methionine supplements, the methylation ratios in the brain and cords of these animals were much closer to normal values. A clue as to how the level of $\mathrm{SAH}$ was being elevated was the finding that in $\mathrm{N}_{2} \mathrm{O}$-treated animals the levels of cystathionine had gone up by several orders of magnitude. Such cystathionine is the first product on the pathway used by cells to degrade homocysteine (Fig. 1). We suggested that what was happening was that as methionine synthase became inactivated during $\mathrm{N}_{2} \mathrm{O}$ treatment the level of methionine in neural tissue would begin to drop. This 
was then compensated for by the increased uptake of plasma methionine across the blood-brain barrier. Such transported methionine could and does maintain the level of methionine and SAM in neural tissues. It would also provide adequate SAM for use by methyltransferases, thus maintaining methylation reactions. However, each such reaction produces $\mathrm{SAH}$ which would normally be recycled back to methionine and SAM via homocysteine. The reduced level of methionine synthase in the pig results in homocysteine accumulating and presumably the degradation pathway to cystathionine and cysteine, while present in pig neural tissue, is not sufficiently active to deal with all the homocysteine so generated. Homocysteine and SAH are kept in enzymic equilibrium by a hydrolase enzyme. This reaction favouring SAH synthesis unless the homocysteine or adenosine are removed. We suggest that in the pig such accumulation occurs reducing the methylation ratio and causing inhibition of methyltransferase. Such inhibition we suggest would in turn cause hypomethylation of myelin basic protein and, perhaps, other constituents of myelin, resulting either in its reduced synthesis or its accelerated degradation which would produce the observed myelopathy. It may be that in animals that do not get the myelopathy on $\mathrm{N}_{2} \mathrm{O}$ treatment such as the rat, that there is a greater capacity to export or degrade homocysteine from the central nervous system. In support of this we have shown that in such animals there is a much smaller reduction in the methylation ratio on $\mathrm{N}_{2} \mathrm{O}$ treatment. It is also possible that their methyltransferases are less sensitive to $\mathrm{SAH}$ inhibition or that hypomethylated myelin in such species is normal enough to maintain adequate neural function.

Does the suggestion that diminished methylation causes the neuropathy fit well with clinical experience? One would not expect folic acid to be successful in treating the neuropathy. The time of presentation of the neuropathy could depend to some extent on protection by dietary methionine. Dentists who abuse $\mathrm{N}_{2} \mathrm{O}$ develop ataxia and a neuropathy identical to that seen in patients with pernicious anaemia (Lazer et al. 1978). Perhaps its most convincing support, however, comes from consideration of a number of patients with a deficiency of 5,10-methylenetetrahydrofolate reductase. As mentioned previously these patients do not get megaloblastic bone marrows or anaemia. However, they do get neurological signs and symptoms similar to those seen in pernicious anaemia. In the seven such patients where post-mortem examinations of their spinal cords were carried out, all had sub-acute combined degeneration of the cord and demyelination of the peripheral nerves as seen in vitamin $B_{12}$ deficiency (Erbe, 1986). In contrast, of patients with a genetic deficiency of the mutase, many were clinically abnormal and mentally retarded but none had the neuropathy associated with vitamin $B_{12}$ deficiency.

\section{REFERENCES}

Amess, J. A. L., Burman, J. F., Rees, G. M., Nancekievill, D. J. \& Mollin, D. L. (1978). Megaloblastic haemopoiesis in patients receiving nitrous oxide. Lancet ii, 339-342.

Baldwin, G. S. \& Carnegie, P. R. (1971). Specific enzymatic methylation of an arginine in the experimental allergic encephalomyelitis protein from human myelin. Science 171, 579-581.

Chanarin, I. (1990). The Megaloblastic Anaemias, 3rd ed. Oxford and London: Blackwell Scientific.

Chanarin, I., Deacon, R., Lumb, M. \& Perry, J. (1980). Vitamin B ${ }_{12}$ regulates folate metabolism by the supply of formate. Lancet ii, 505-507.

Deacon, R., Lumb, M., Perry, J., Chanarin, J., Minty, B., Halsey, M. \& Nunn, J. (1980). Inactivation of methionine synthase by nitrous oxide. European Journal of Biochemistry 104, 419-422. 
Dinn, J. J., Weir, D., McCann, S., Reed, B., Wilson, P. \& Scott, J. M. (1980). Methyl group deficiency in nerve tissue: A hypothesis to explain the lesion of subacute combined degeneration. Irish Journal of Medical Science 149, 1-4.

Erbe, R. W. (1986). Inborn errors of folate metabolism. Folates and Pterins, vol. 3, pp. 413-465 [R. L. Blakley and V. M. Whitehead, editors]. New York: John Wiley and Sons.

Frenkel, R. P. (1973). Abnormal fatty acid metabolism: peripheral nerves of patients with pernicious anaemia. Journal of Clinical Investigation 52, 1237-1245.

Herbert, V. \& Zalushy, R. (1962). Interrelation of vitamin $B_{12}$ and folic acid metabolism: Folic acid clearance studies. Journal of Clinical Investigation 41, 1263-1276.

Hoffman, D. R., Marion, D. W., Cornatzer, W. E. \& Duerre, J. A. (1980). S-adenosylmethionine and S-adenosylhomocysteine metabolism in isolated rat liver. Journal of Biological Chemistry 255, 822-827.

Keating, S., Weir, D. G. \& Scott, J. M. (1983). Factors affecting folate polyglutamate biosynthesis in rat liver. In Chemistry and Biology of Pterdines, pp. 977-981 [J. A. Blair, editor]. Berlin and New York: Walter de Gruyter.

Kutzbach, C. \& Stokstad, E. L. R. (1967). Feedback inhibition of methylene-tetrahydrofolate reductase in rat liver by S-adenosylmethionine. Biochemica et Biophysica Acta 139, 217-220.

Lazer, R. N., Fishman, R. A. \& Schaler, J. A. (1978). Neuropathy following abuse of nitrous oxide. Neurology 28, 504-506.

Lumb, M., Sharer, N., Deacon, R., Jennings, P., Purkiss, P., Perry, J. \& Chanarin, I. (1983). Effects of nitrous oxide induced inactivation of cobalamin on methionine and $\mathrm{S}$-adenosylmethionine metabolism in the rat. Biochemica et Biophysica Acta 756, 354-359.

McGing, P., Reed, B., Weir, D. G. \& Scott, J. M. (1978). The effect of vitamin $B_{12}$ inhibition in vivo: Impaired folate polyglutamate biosynthesis indicating that 5-methyltetrahydropteroylglutamate is not its usual substrate. Biochemical and Biophysical Research Communications 82, 540-546.

McKeever, M. P., Weir, D. G., Molloy, A. \& Scott, J. M. (1991). Betaine-homocysteine methyltransferase: Organ distribution in man, pig and rat and subcellular distribution in the rat. Clinical Sciences (In the Press).

Scott, J. M., Dinn, J. J., Wilson, P. \& Weir, D. G. (1981). Pathogenesis of subacute combined degeneration. A result of methyl group deficiency. Lancet ii, 334-337.

Scott, J. M., Reed, B., McKenna, B., McGing, P., McCann, S., O'Sullivan, H., Wilson, P. \& Weir, D. G. (1979). A study of the multiple changes induced in vivo in experimental animals by inactivations of vitamin $\mathrm{B}_{12}$ using nitrous oxide. Chemistry and Biology of Pterdines. Developments in Biochemistry, vol. 4, pp. 335-340 [R. L. Kislink and G. M. Brown, editors]. Amsterdam: Elsevier North Holland.

Scott, J. M. \& Weir, D. G. (1981). The methyl folate trap. A physiological response in man to prevent methyl group deficiency in kwashiorkor (methionine deficiency) and an explanation for folic acid-induced exacerbation of sub-acute combined degeneration. Lancet ii, 337-340.

Van der Westhuyzen, J. \& Metz, J. (1983). Tissue S-adenosylmethionine levels in fruit bats Rousettus aegyptiacus with nitrous oxide-induced neuropathy. British Journal of Nutrition 50, 325-330.

Weir, D. G., Keating, S., Molloy, A., McPartlin, J., Kennedy, S., Blanchflower, J., Kennedy, D. G., Rice, D. \& Scott, J. M. (1988). Methylation deficiency causes vitamin $B_{12}$-associated neuropathy in the pig. Journal of Neurochemistry 51, 1949-1952. 